\title{
Therapeutic mechanisms of ibuprofen, prednisone and betamethasone in osteoarthritis
}

\author{
FENGLONG SUN, YAOHUA ZHANG and QIANG LI
}

Department of Orthopedics, Beijing Rehabilitation Hospital, Capital Medical University, Beijing 100144, P.R. China

Received November 3, 2015; Accepted November 10, 2016

DOI: $10.3892 / \mathrm{mmr} .2016 .6068$

\begin{abstract}
The present study aimed to investigate the therapeutic mechanisms of nonsteroidal anti-inflammatory drugs (NSAIDs) and steroids in osteoarthritis (OA). The CHON-002 human chondrocyte cell line was used in the study. The levels of the cytokines, interleukin (IL)-1 $\beta$, IL-6, IL- 8 and IL-10, released by cells treated with tumor necrosis factor- $\alpha$ (TNF- $\alpha$ ) were determined by ELISA. Levels of collagen I, aggrecan, matrix metalloproteinase (MMP)-1, MMP-13, signal transducer and activator of transcription (STAT) 3, nuclear factor- $\kappa \mathrm{B}(\mathrm{NF}-\kappa \mathrm{B})$ subunit p65 and inhibitory subunit of NF- $\kappa \mathrm{B}(\mathrm{I} \kappa \mathrm{B})$ following treatment with IL-1 $\beta$, IL-6, IL- 8 or IL-10 were assessed by western blot. Levels of IL- 6 and IL- 8 were measured by ELISA following administration of TNF- $\alpha$ combined with certain drugs. In addition, these parameters were evaluated by western blot following incubation with drugs in combination with IL-6 or IL-8 and after knockdown of STAT3, by addition of small interfering RNA (siRNA)-STAT3 (siSTAT3), an inhibitor of the proteasome (MG132) or both. IL-1 $\beta$, IL-6, IL-8 and IL-10 were upregulated by TNF- $\alpha$. Addition of IL- 6 or IL- 8 led to increased collagen I, MMP-1 and MMP-13 protein levels, and also promoted STAT3 phosphorylation and increased the expression of NF- $\mathrm{B}$ subunit $\mathrm{p} 65$, but had no effect on aggrecan protein levels. When siSTAT3 and MG132 treatment was combined, levels of collagen I, MMP-1 and MMP-13 were reduced. Additionally, levels of IL- 6 and IL- 8 were significantly decreased by prednisone, ibuprofen and betamethasone. However, no significant differences were observed following treatment with piroxicam or indomethacin. In combination with IL-6 or IL-8, prednisone, ibuprofen and betamethasone significantly reduced the levels of collagen I, MMP-1 and MMP-13, and inactivated NF- $\mathrm{NB}$ and STAT3 pathways. In conclusion, prednisone, ibuprofen and betamethasone may prevent OA by suppressing the expression of IL-6 and IL-8,
\end{abstract}

Correspondence to: Dr Fenglong Sun, Department of Orthopedics, Beijing Rehabilitation Hospital, Capital Medical University, 32 Badachu, Shijingshan, Beijing 100144, P.R. China

E-mail: sunfenglong3369@126.com

Key words: osteoarthritis, ibuprofen, prednisone, betamethasone, interleukin-6, interleukin-8 subsequently inactivating NF- $\kappa \mathrm{B}$ and STAT3 pathways, and ultimately, leading to decreased levels of collagen I, MMP-1, and MMP-13.

\section{Introduction}

Osteoarthritis (OA) is a common degenerative disorder of joints characterized by the progressive breakdown of articular cartilage. It is a global public health problem that causes substantial pain and disability in middle-aged and older individuals, and leads to an economic burden and a reduced quality of life $(1,2)$. However, no curative therapeutics are currently available for OA. Currently, the standard treatment for OA focuses on the control of pain and improvement of joint function. The most commonly prescribed medications include locally administered corticosteroids (e.g., prednisone and betamethasone), nonsteroidal anti-inflammatory drugs (NSAIDs; e.g., piroxicam, ibuprofen and indomethacin) and other anti-inflammatory drugs. It is well established that the development and progression of OA are associated with inflammation even in the early phase of the disease $(3,4)$. Inflammatory molecules, including pro-inflammatory cytokines, are reported to be important mediators involved in the pathophysiology of OA (5). Tumor necrosis factor (TNF)- $\alpha$ and interleukin (IL)-1 $\beta$ are the major pro-inflammatory cytokines that lead to significant breakdown of the cartilage macromolecules $(5,6)$. They act independently or in conjunction with other cytokines to initiate inflammation. Previous studies have demonstrated that TNF- $\alpha$ and IL-1 $\beta$ directly downregulate the expression of extracellular matrix components, including aggrecan and type II collagen $(7,8)$ and increase the expression of catabolic factors, including matrix metalloproteinase (MMP)-1 and MMP-13 $(9,10)$. IL-10 protects cartilage, and stimulates the expression of type II collagen and proteoglycan $(11,12)$. It reverses the damaging effects of TNF- $\alpha$ and IL-1 $\beta$ on cartilage by inhibiting the production of MMPs, pro-inflammatory cytokines and nitric oxide (13). IL-6 is known to have pro-inflammatory and anti-inflammatory effects. IL- 6 release is stimulated by TNF- $\alpha$ and it is thought to upregulate the number of inflammatory cells in synovial tissue (14). However, other studies have suggested that IL-6 negatively regulates further expression of $\mathrm{TNF}-\alpha$ (14). In addition to the aforementioned pro-inflammatory cytokines, chemokines are also implicated in OA (5). IL-8 is a chemokine that is synthesized in OA chondrocytes and has an important 
role in the pathogenesis of OA. It promotes the production of MMP-1 and MMP-13 by articular chondrocytes (15). However, limited information is available regarding studies on the therapeutic mechanisms of corticosteroids and NSAIDs in OA. Therefore, the aim of the current study was to identify the potential underlying mechanisms. The present study employed prednisone, betamethasone, piroxicam, ibuprofen and indomethacin to investigate therapeutic mechanisms in OA. The findings of the present study may help to guide the clinical management of OA.

\section{Materials and methods}

Cell culture. CHON-002 human chondrocyte cell line, derived from the long bone of an 18-week-old female fetus, was purchased from the American Type Culture Collection (Manassas, VA, USA). The CHON-002 cell line was cultured in Dulbecco's modified Eagle's medium (Thermo Fisher Scientific, Inc., Waltham, MA, USA) supplemented with $0.1 \mathrm{mg} / \mathrm{ml} \mathrm{G}-418,10 \%$ fetal bovine serum (FBS; Thermo Fisher Scientific, Inc.), $100 \mathrm{U} / \mathrm{ml}$ penicillin (Thermo Fisher Scientific, Inc.) and $100 \mathrm{mg} / \mathrm{ml}$ streptomycin (Thermo Fisher Scientific, Inc.) in a humidified atmosphere of $5 \% \mathrm{CO}_{2}$ at $37^{\circ} \mathrm{C}$.

Drug preparation and treatment. All drugs (piroxicam, ibuprofen, indomethacin, prednisone and betamethasone) were purchased from Sigma-Aldrich; Merck Millipore (Darmstadt, Germany) as powders. Drugs were prepared as the following treatment groups: Piroxicam $(5 \mu \mathrm{M})$, ibuprofen $(400 \mu \mathrm{M})$, indomethacin $(100 \mu \mathrm{M})$, prednisone $(5 \mu \mathrm{M})$ and betamethasone $(10 \mu \mathrm{M})$. Dimethyl sulfoxide $(10 \mu \mathrm{l})$ was applied to fully dissolve $1 \mathrm{mg}$ of each drug. Subsequently, the dissolved mixture was diluted with serum-free F12K media (Sigma-Aldrich; Merck Millipore) supplemented with $5 \mathrm{ml}$ penicillin, $5 \mathrm{ml}$ streptomycin and $50 \mu \mathrm{g} / \mathrm{ml}$ ascorbic acid. Additionally, all cytokines (TNF- $\alpha$, IL-1 $\beta$, IL-6, IL-8 and IL-10) were obtained in lyophilized powdered form from R\&D Systems, Inc. (Minneapolis, MN, USA) and dissolved in sterile $4 \mathrm{mM}$ hydrochloric acid supplemented with $1 \mathrm{mg} / \mathrm{ml}$ bovine serum albumin. Cytokines were prepared as the following treatment groups: TNF- $\alpha(5 \mathrm{ng} / \mathrm{ml})$, IL-1 $\beta(1 \mathrm{ng} / \mathrm{ml})$, IL-6 $(10 \mathrm{ng} / \mathrm{ml})$, IL-8 $(10 \mathrm{ng} / \mathrm{ml})$ and IL-10 $(5 \mathrm{ng} / \mathrm{ml})$. In addition, an inhibitor of the proteasome, MG132 $(10 \mu \mathrm{M}$; Selleck Chemicals, Inc., Houston, TX, USA) was added to inhibit NF- $\kappa \mathrm{B}$ by preventing the degradation of the inhibitory subunit of NF- $\kappa \mathrm{B}(\mathrm{I} \kappa \mathrm{B} \alpha)$.

Cell viability assay. Cells were plated onto 96-well plates at a final concentration of $1 \times 10^{4}$ cells/well in culture medium with drugs (piroxicam, ibuprofen, indomethacin, prednisone and betamethasone) or cytokines (TNF- $\alpha$, IL-1 $\beta$, IL-6, IL-8 and IL-10). Untreated cells were considered as controls. At $48 \mathrm{~h}$ after treatment, $5 \mathrm{mg} / \mathrm{ml}$ MTT (20 $\mu \mathrm{l}$; Sigma-Aldrich; Merck Millipore) was added to each well, followed by incubation for $4 \mathrm{~h}$ at $37^{\circ} \mathrm{C}$. Thereafter, dimethylsulfoxide (DMSO; Sigma-Aldrich; Merck Millipore) was added to dissolve the formazan. Absorbance at $570 \mathrm{~nm}$ was measured using a Synergy plate reader (BioTek Instruments, Inc., Winooski, VT, USA).

ELISA. After $48 \mathrm{~h}$ of treatment, the conditioned medium was collected forELISA.Levels of IL-1 $\beta$,IL-6,IL-8 and IL-10 in cells with or without TNF- $\alpha$ treatment, and levels of IL-6 and IL-8 in cells with or without TNF- $\alpha$ treatment, drugs (piroxicam, ibuprofen, indomethacin, prednisone and betamethasone) or TNF- $\alpha$ in combination with drugs were respectively determined by human IL-1 $\beta$ ELISA Kit (cat. no. CSB-E08053h), human IL-6 ELISA Kit (cat. no. CSB-E04638h), human IL-8 ELISA Kit (cat. no. CSB-E04641h) and human IL-10 ELISA Kit (cat. no. CSB-E04593h) according to the manufacturer's protocol. All ELISA kits were purchased from Flarebio Biotech LLC (College Park, MD, USA).

Small interfering RNA (siRNA) transfection. Cells in the exponential growth phase were seeded in a $60 \mathrm{~mm}$ dish at a concentration of $5 \times 10^{5}$ cells/well. Transfection was performed when cells reached $70 \%$ confluence. Signal transducer and activator of transcription (STAT) 3 was silenced through the use of an siRNA targeting STAT3 (siSTAT3). siSTAT3 and negative control siRNA-were designed and constructed by Shanghai GenePharma Co., Ltd. (Shanghai, China). The targeting sequence for siSTAT3 was 5'-CAGGCTGGTAAT TTATATAAT-3' and negative control siRNA was 5'-CATTGA CTTATAAATTCGTTC-3'. After $24 \mathrm{~h}$ of incubation, cells were transfected using Lipofectamine ${ }^{\circledR} 2000$ (reagent (Thermo Fisher Scientific, Inc.) according to the manufacturer's instructions. At $48 \mathrm{~h}$ after the transfection, the cells were prepared for western blot analysis.

Protein isolation and western blot analysis. Following stimulation with or without cytokines or drugs, total cellular protein and nuclear protein were extracted from the cells using nuclear and cytoplasmic extraction reagent kits (Beyotime Institute of Biotechnology, Inc., Haimen, China) and stored at $-80^{\circ} \mathrm{C}$ until use. Protein levels of STAT3, phosphorylated STAT3 (pSTAT3 ${ }^{\text {Ser727 }}$ and pSTAT3 ${ }^{\text {Tyr705}}$ ), collagen I, MMP-1, MMP-13, aggrecan and I $\mathrm{B}$ were assessed using total cellular protein, while protein levels of $\mathrm{NF}-\kappa \mathrm{B}$ subunit $\mathrm{p} 65$ were assessed using total cellular protein and nuclear protein. The protein concentration was quantified using a bicinchoninic acid protein assay kit (Pierce; Thermo Fisher Scientific, Inc.). Protein samples (20 $\mu \mathrm{g}$ per lane) were resolved on a 10-12\% SDS-PAGE gel and blotted onto PVDF or nitrocellulose membranes, blocked in $5 \%$ fresh non-fat milk in phosphate buffered saline for $2 \mathrm{~h}$ at room temperature and probed with primary antibodies against pSTAT3 $3^{\text {Ser727 }}$ (cat. no. 9134; 1:1,000; Cell Signaling Technology, Inc.,

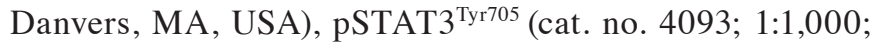
Cell Signaling Technology, Inc.), STAT3 (cat. no. 12640; 1:1,000; Cell Signaling Technology, Inc.), collagen I (cat. no. sc-29009; 1:1,000; Santa Cruz Biotechnology, Inc., Dallas, TX, USA), MMP-1 (cat. no. sc-137044; 1:1,000; Santa Cruz Biotechnology, Inc.), MMP-13 (cat. no. sc-12363; 1:1,000; Santa Cruz Biotechnology, Inc.), aggrecan (cat. no. sc-70332; 1:1,000; Santa Cruz Biotechnology, Inc.), IкB (cat. no. BS2224; 1:1,000; Bioworld Technology, Inc., St. Louis Park, MN, USA), and p65 (cat. no. 8242; 1:1,000; Cell Signaling Technology, Inc.) overnight at $4^{\circ} \mathrm{C}$. Lamin B (cat. no. 12255; 1:1,000; Cell Signaling Technology, Inc.) was used as an internal nuclear protein loading control, while GAPDH (cat. no. 5174; 1:1,000; Cell Signaling Technology, Inc.) was used as an internal total protein loading control. 
A
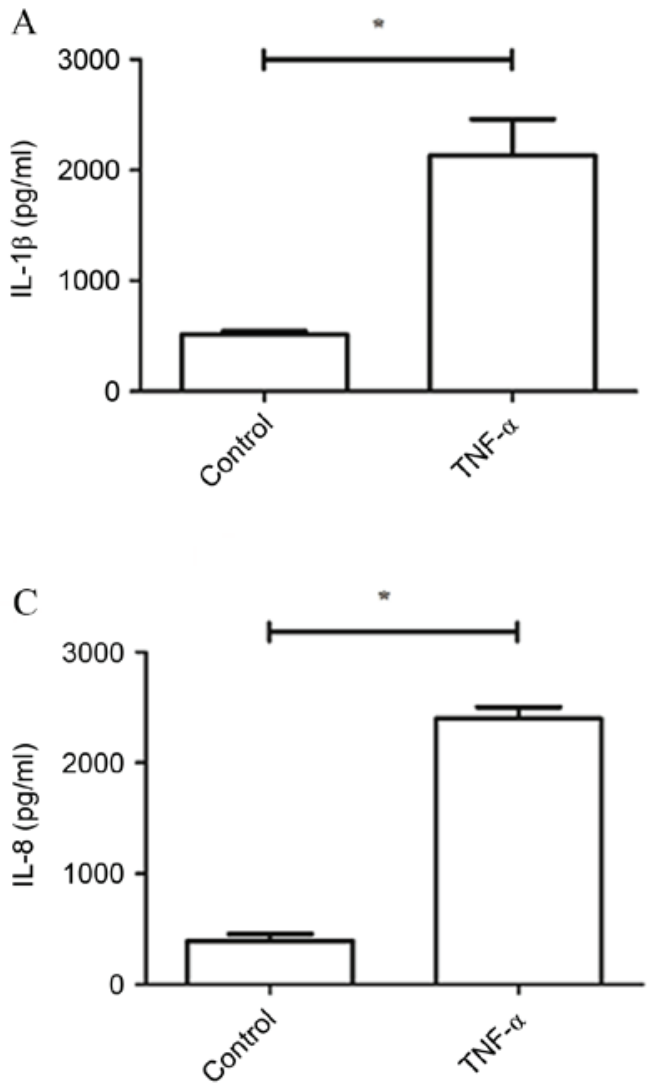

B

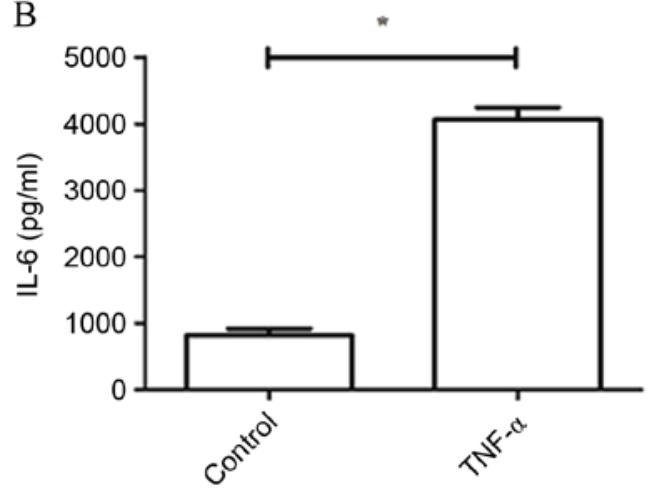

D

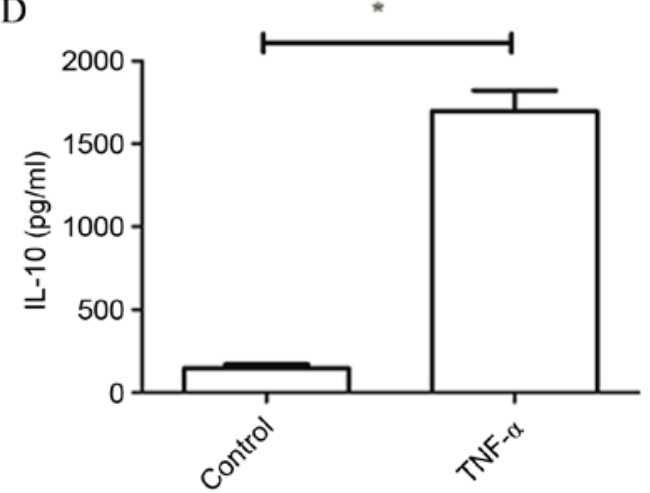

Figure 1. TNF- $\alpha$ increases the expression levels of IL-1 $\beta$, IL-6, IL-8 and IL-10. Expression levels of (A) IL-1 $\beta$, (B) IL-6, (C) IL-8 and (D) IL-10 following stimulation by TNF- $\alpha$ compared with control levels. ${ }^{*} \mathrm{P}<0.05$ vs. control. TNF, tumor necrosis factor; IL, interleukin.

The membranes were then incubated with secondary IgG conjugated to horseradish peroxidase (cat. no. 7071; 1:5,000; Cell Signaling Technology, Inc.) for $1 \mathrm{~h}$ at room temperature. Immunoreactive protein bands were visualized by enhanced chemiluminescence western blotting substrate (Pierce; Thermo Fisher Scientific, Inc.). The immunoreactive bands were analyzed using Image Gauge version 4.0 software (Fujifilm, Tokyo, Japan). Each condition was carried out in triplicate.

Statistical analysis. Data are expressed as the mean \pm standard deviation. SPSS version no. 17.0 software (SPSS, Inc., Chicago, IL, USA) was used for statistical analysis. Student's t-test was performed to calculate P-values for 2 groups, or one-way analysis of variance followed by Tukey-Kramer's post hoc test for multiple comparisons. $\mathrm{P}<0.05$ was considered to indicate a statistically significant difference.

\section{Results}

Expression levels of $I L-1 \beta, I L-6, I L-8$ and $I L-10$ with or without TNF- $\alpha$ treatment. The present study used doses of TNF- $\alpha(5 \mathrm{ng} / \mathrm{ml})$ to activate cultured chondrocytes and to stimulate an inflammatory response in vitro. Primary cultures that were not treated with TNF- $\alpha$ served as a control group. Levels of IL-1 $\beta$, IL-6, IL-8 and IL-10 were determined by ELISA. Compared with the control group, expression levels of IL-1 $\beta$, IL-6, IL- 8 and IL-10 were all significantly increased by TNF- $\alpha(\mathrm{P}<0.05$; Fig. 1$)$.
Expression of collagen I, aggrecan, MMP-1 and MMP-13 with or without cytokines. Western blotting was performed to observe the effects of cytokines, including IL-1 $\beta$, IL-6, IL-8 and IL-10, on the protein expression of collagen I, aggrecan, MMP-1 and MMP-13. The results demonstrated that expression levels of collagen I, MMP-1 and MMP-13 were all markedly increased by treatment with IL- 6 or IL-8. However, there were no observable differences in protein levels of aggrecan when treated with IL-1 $\beta$, IL-6, IL-8 or IL-10 compared with the control group. Although expression levels of collagen I, MMP-1 and MMP-13 were elevated by treatment with IL-1 $\beta$ or IL-10, there was no statistically significant difference between the experimental and the control groups (Fig. 2A).

Promotion of STAT3 phosphorylation and activation of $N F-\kappa B$ subunit $p 65$ by IL- 6 and $I L-8$. To investigate the associations between the STAT3 or NF- $\mathrm{B}$ p65 signaling pathways and IL- 6 and IL-8, the present study determined the levels of pSTAT3 $^{\text {Ser727 }}$, pSTAT3 $^{\text {Tyr705 }}$, STAT3, IкB and p65 following stimulation with IL-6 or IL-8. As presented in Fig. 2B, the expression levels of pSTAT3 ${ }^{\text {Ser727 }}$ and pSTAT3 $^{\text {Tyr705 }}$ were visibly increased by treatment with IL-6 or IL-8 compared with the control group, indicating that STAT3 phosphorylation was promoted by IL-6 and IL-8. Additionally, following treatment with IL- 6 or IL-8, the expression of I $\mathrm{B}$ was lower than the control group, while the expression of p65 (both in the cell nucleus and whole cell) was higher than the control group, suggesting that $N F-\kappa B$ p 65 was activated by stimulation by IL-6 or IL-8. 
B

A

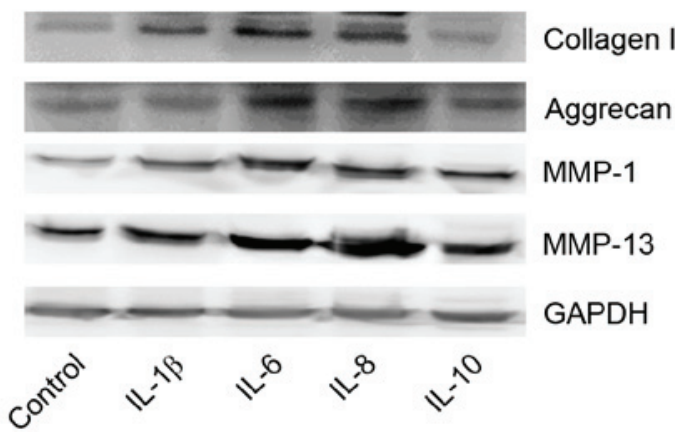

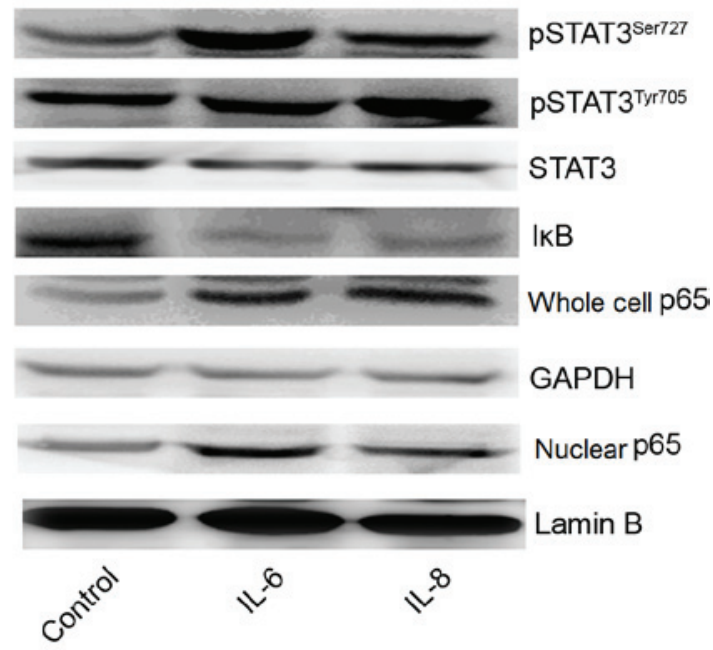

Figure 2. Effects of cytokines on expression of collagen I, aggrecan, MMP-1, MMP-13, STAT3 and NF- $\mathrm{B}$ p65 signaling pathways. (A) Effects of IL-1 3 , IL-6, IL-8 and IL-10 on expression of collagen I, aggrecan, MMP-1 and MMP-13. (B) Effects of IL-6 and IL-8 expression on STAT3 and NF- $\mathrm{kB}$ p65 signaling

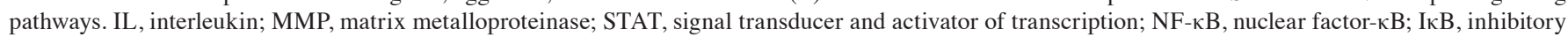
subunit of NF-кB; pSTAT3, phosphorylated STAT3.

A

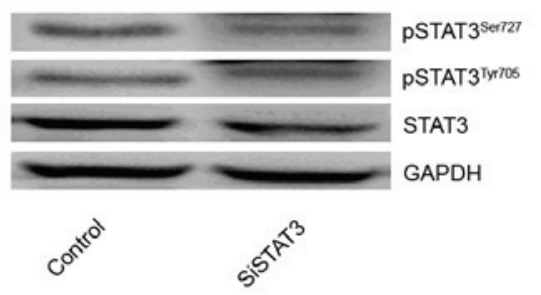

B

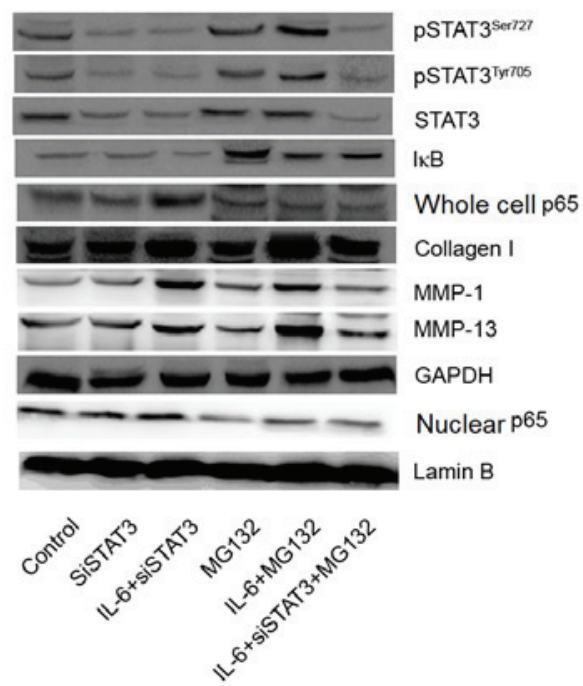

$\mathrm{C}$

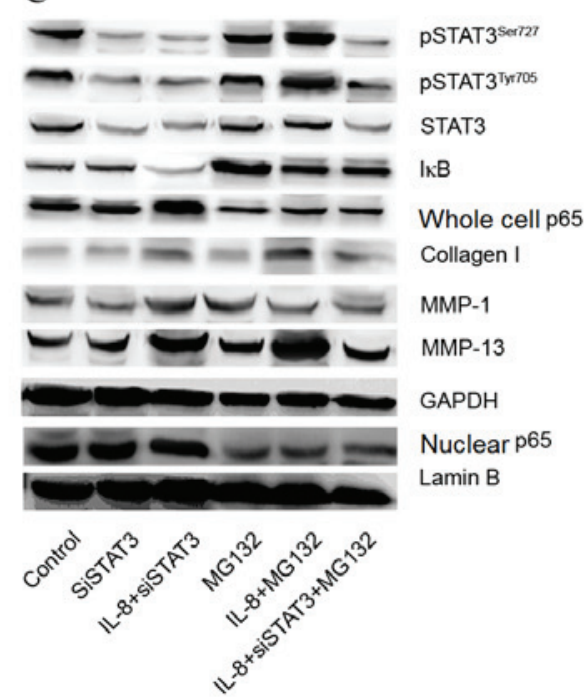

Figure 3. Effects of STAT3 and NF-кB inhibition on expression of collagen I, aggrecan, MMP-1 and MMP-13. (A) Effects of siSTAT3 on expression of STAT3. (B) Effects of IL-6 combined with STAT3 and NF-kB inhibition (using MG132) on the expression of collagen I, aggrecan, MMP-1 and MMP-13. (C) Effects of IL-8 combined with STAT3 and NF-kB inhibition on expression of collagen I, aggrecan, MMP-1 and MMP-13. IL, interleukin; MMP, matrix metal-

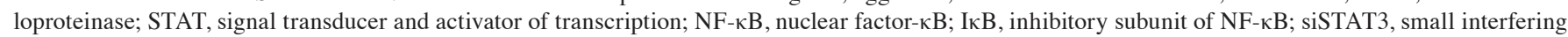
RNA-STAT3; pSTAT3, phosphorylated STAT3.

Effects of STAT3 and NF- $\mathrm{B} B$ inhibition on expression of collagen I, aggrecan, MMP-1 and MMP-13. To investigate whether IL-6 and IL-8 affect the expression of collagen I, MMP-1, and MMP-13 via STAT3 and NF- $\mathrm{BB}$ p65 signaling pathways, the expression of STAT3 was silenced and NF- $\mathrm{KB}$ was inhibited by use of MG132. As shown in Fig. 3A, the expression of pSTAT3 $^{\text {Ser727 }}$ and pSTAT3 $^{\text {Tyr705 }}$ were visibly decreased following transfection with siSTAT3 compared with the control. Following siSTAT3 transfection, the effects of IL-6 and IL-8 on the expression of collagen I, MMP-1 and MMP-13 were decreased, but the effects were not completely eliminated, indicating that other mechanisms exist for the regulation of collagen I, MMP-1 and MMP-13 expression. However, expression of collagen I, MMP-1 and MMP-13 was reduced when treated with the MG132 and transfected with siSTAT3 compared with the IL-6/IL-8+ siSTAT3 group (Fig. 3B and C). The results demonstrated that the effects of IL-6 and IL-8 on the expression of collagen I, MMP-1, and MMP-13 may be through promotion of STAT3 phosphorylation and activation of NF-кB p65.

Effects of drugs and cytokines on cell viability and expression of IL- 6 and IL-8. Compared with the control group, no change in cell viability was observed following treatment with cytokines 
A
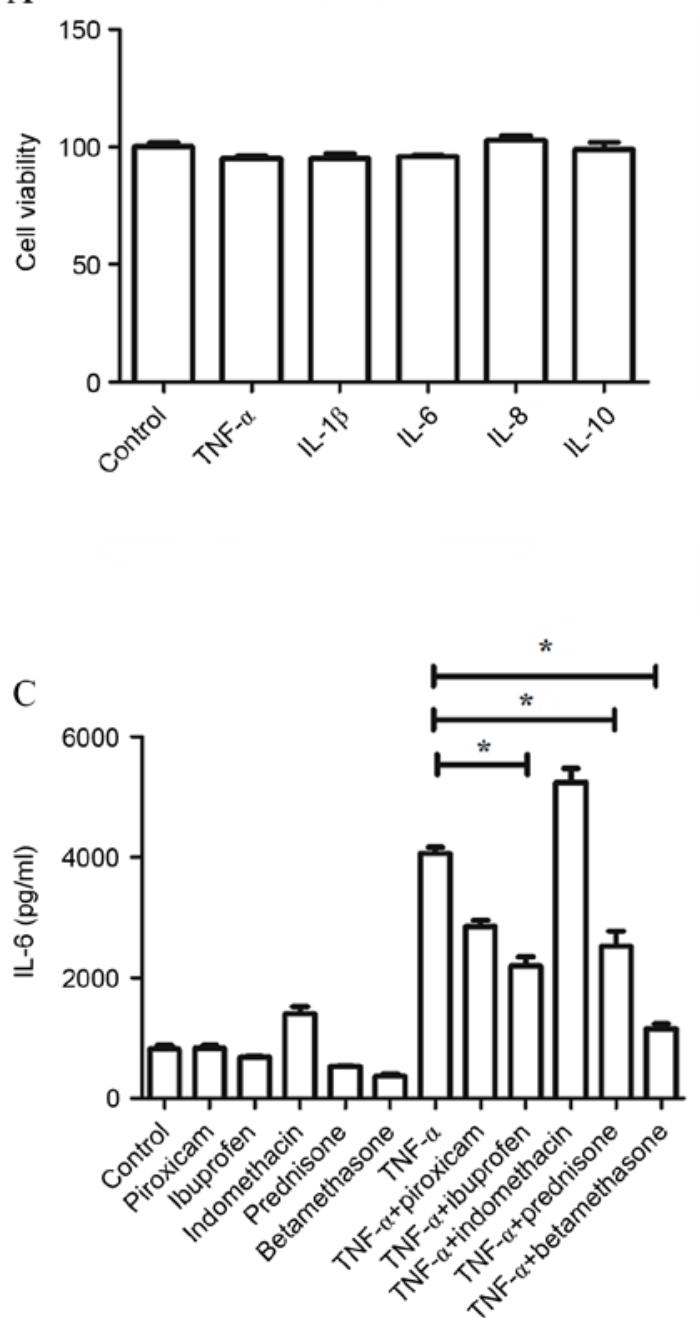

B
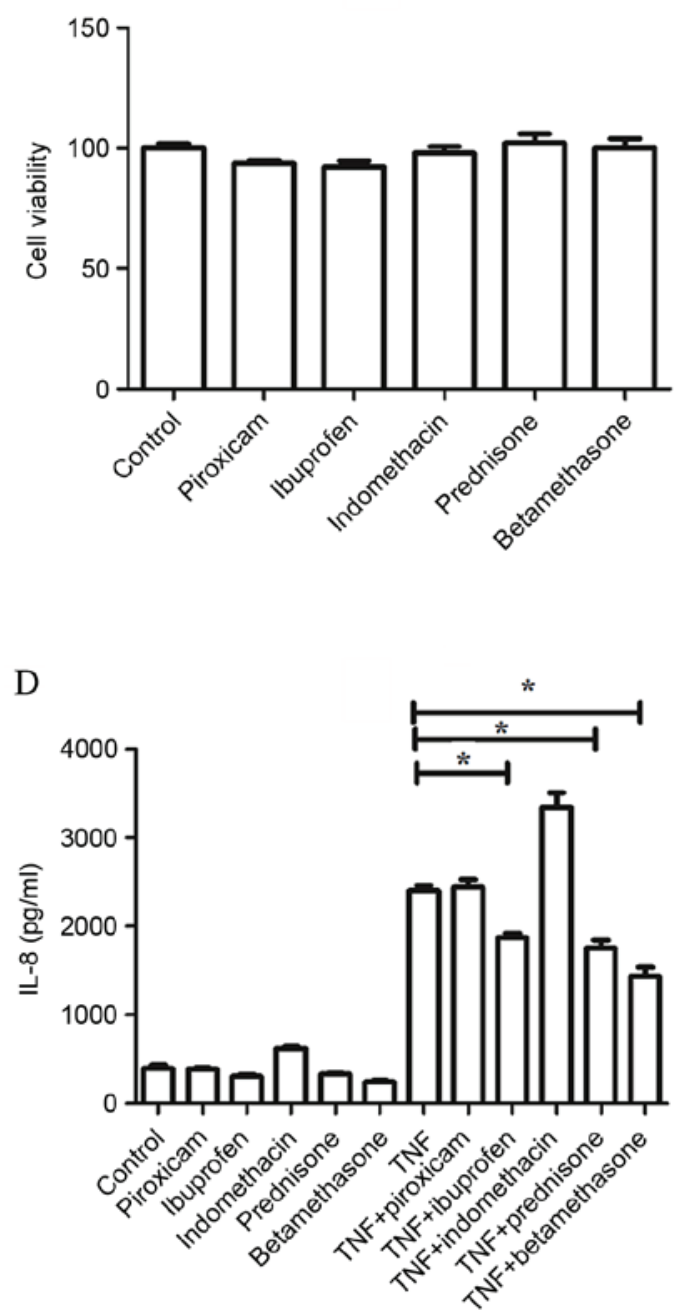

Figure 4. Effects of drugs and cytokines on cell viability and expression of IL-6 and IL-8. (A) Effects of cytokines on cell viability. (B) Effects of drugs on cell viability. (C) Effects of drugs and TNF- $\alpha$-drug combinations on IL- 6 expression. (D) Effects of drugs and TNF- $\alpha$-drug combinations on IL- 8 expression. Comparison indicated by brackets on figure. ${ }^{*} \mathrm{P}<0.05$. TNF, tumor necrosis factor; IL, interleukin.

(TNF- $\alpha$, IL-1 $\beta$, IL-6, IL-8 and IL-10) or drugs (piroxicam, ibuprofen, indomethacin, prednisone and betamethasone). There were no statistically significant differences in cell viability between groups, and the average cell viability was $>90 \%$ (Fig. 4A and B). Additionally, no significant differences were observed in the expression of IL- 6 and IL- 8 measured by ELISA following treatment with drugs alone. However, the increased levels of IL- 6 and IL- 8 induced by TNF- $\alpha$ were significantly decreased when combined with administration of prednisone, ibuprofen or betamethasone $(\mathrm{P}<0.05)$, whereas no significant differences were found between the levels of IL-6 and IL- 8 following stimulation by TNF- $\alpha$ alone compared with TNF- $\alpha$ combined with administration of piroxicam or indomethacin (Fig. 4C and D).

Effect of IL-6 or IL-8 combined with drugs on STAT3, NF-kB p65, collagen I, MMP-1 and MMP-13 expression. To investigate the effects of drugs on expression of STAT3 and NF-kB p65 signaling pathways, as well as the production of Collagen I, MMP-1 and MMP-13, we exposed the cells to drug and IL- 6 or IL- 8 combinations. The present study observed that the expression of pSTAT3 $3^{\text {Ser727 }}$, pSTAT3 ${ }^{\text {Tyr705 }}$, p65 (both in the cell nucleus and whole-cell), collagen I, MMP-1 and MMP-13 were reduced, while the levels of $\mathrm{I} \kappa \mathrm{B}$ were increased by administration of prednisone, ibuprofen and betamethasone. These results suggested that prednisone, ibuprofen and betamethasone decreased the inflammatory response and prevented abnormal catabolism (Fig. 5A and B).

\section{Discussion}

The present study demonstrated that cultured chondrocytes are activated by TNF- $\alpha$, which leads to increased expression of IL-1 $\beta$, IL- 6 , IL- 8 and IL-10, which are associated with OA. Of the cytokines investigated in the present study, IL-6 and IL- 8 increased the levels of collagen I, MMP-1 and MMP-13, promoted STAT3 phosphorylation and activated NF- $\mathrm{NB}$ p65. Additionally, corticosteroids (prednisone and betamethasone) and NSAIDs (ibuprofen) reduced the expression of IL-6 and IL-8, inactivated STAT3 and NF- $\kappa$ B signaling pathways, and reduced the expression of collagen I, MMP-1 and MMP-13, which protect against OA. In order to observe the efficacy of NSAIDs and steroids in preventing OA, TNF- $\alpha$, at an optimized concentration of $5 \mathrm{ng} / \mathrm{ml}$, was 
A

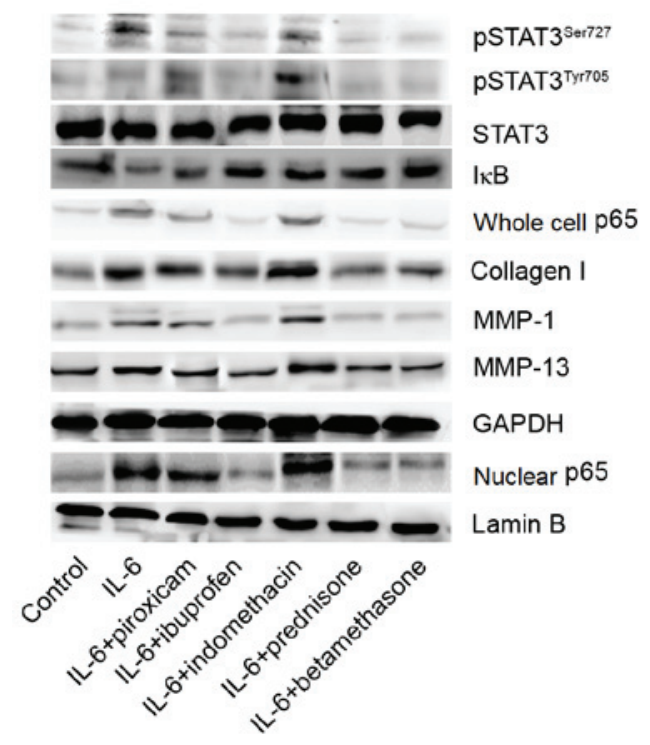

B

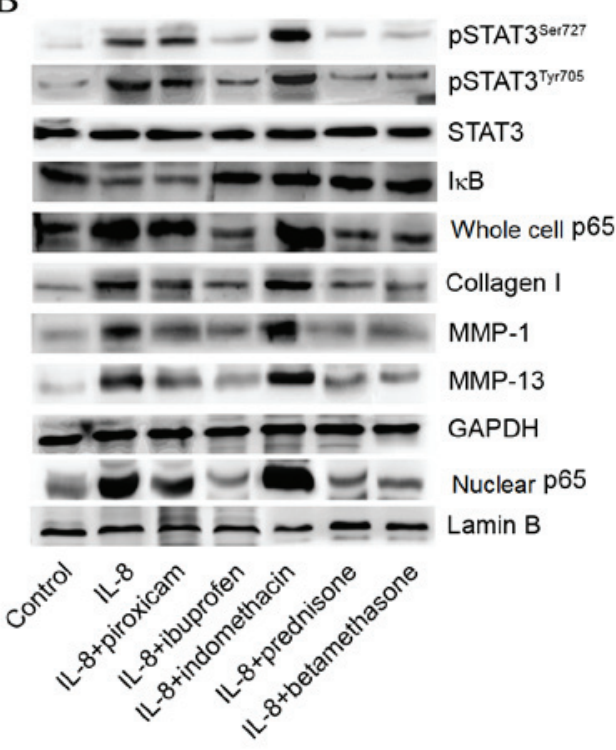

Figure 5. Effects of IL-6-drug and IL-8-drug combinations on STAT3, NF- $\mathrm{B}$ p65, collagen I, MMP-1 and MMP-13 expression. (A) Effect of IL-6-drug combinations on STAT3, NF-кB p65, collagen I, MMP-1 and MMP-13 expression. (B) Effect of IL-8 plus drugs on STAT3, NF-кB p65, collagen I, MMP-1 and MMP-13 expression. IL, interleukin; MMP, matrix metalloproteinase; STAT, signal transducer and activator of transcription; NF- $\mathrm{B}$, nuclear factor-kappa B;

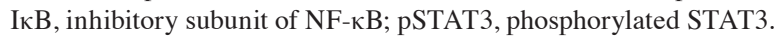

used to stimulate the cultured chondrocytes and to mimic a catabolic environment in vitro in the present study $(16,17)$. The role of pro-inflammatory mediators in the pathophysiology of OA has been extensively investigated $(4,17,18)$. An improved understanding of the role of cytokines involved in the pathophysiology of OA is important for the identification of potential therapeutic targets. Pro-inflammatory cytokines and chemokines locally produced by chondrocytes contribute to the pathological processes in OA. TNF- $\alpha$, IL-1 $\beta$, IL-6, IL- 8 and IL-10 enhance inflammation of the joints and subsequently lead to the degradation of cartilage (5). It has been reported that TNF- $\alpha$ may induce the production of IL-6 (19), IL-8 (20) and IL-10 (21). Corresponding with previous studies, the present study also observed that chondrocytes were activated by TNF- $\alpha$. Expression levels of IL-6, IL- 8 and IL-10 were all significantly increased by TNF- $\alpha$ addition. In addition, the levels of IL- $1 \beta$ were also increased by TNF- $\alpha$. Elevated IL-6 and IL-8 levels have been previously described in the serum and synovial fluid of patients with OA $(22,23)$. When combined with IL-1 $\beta$, the expression of type II collagen was suppressed and the release of MMP-1 and MMP-13 was stimulated by IL-6 (24,25). Furthermore, the production of tissue inhibitors of metalloproteinases can be induced by IL-6, thereby, suppressing proteolytic damage induced by MMPs (14). Additionally, animal experimental studies have suggested that IL-6-deficient mice can spontaneously develop $\mathrm{OA}$ and that the cartilage repair response is damaged in these mice (26). IL-8, also known to be an osteoclastogenic factor, is responsible for osteoclastogenesis and the bone resorption process, which is involved in the OA cartilage degradation process (27). Furthermore, IL- 8 promotes the activation of mononuclear cell leukocytes into the synovium, degranulation of neutrophils, production of MMP-13 by chondrocytes, apoptosis of chondrocytes and loss of proteoglycans (28-30). The present study observed that IL- 6 and IL- 8 elevated the levels of collagen I, MMP-1 and MMP-13, and also increased STAT3 phosphorylation and NF- $\mathrm{B}$ p65 levels. Previous studies have demonstrated that NF- $\kappa \mathrm{B}$ (p65/p50), NF- $\kappa \mathrm{B} / \mathrm{I} \kappa \mathrm{B}$ and STAT3 signaling are abnormally activated in OA chondrocytes $(4,31,32)$. The $N F-\kappa B$ pathway is an essential regulator of the inflammatory cytokine-induced catabolic response in chondrocytes. Activation of $\mathrm{NF}-\kappa \mathrm{B}$ (p65/p50) signaling is vital to allow chondrocytes to induce production of MMPs, nitric oxide synthase 2, cyclooxygenase-2 and IL-1 (4). NF- $\kappa \mathrm{B}$ mediates the expression of pro-inflammatory cytokines including TNF- $\alpha$, IL-1 $\beta$ and IL-6, and chemokines (33). Latourte et al (34) suggested that IL-6 has various catabolic effects on cartilage, predominantly regulated by STAT3. Blockade of STAT3 protected against OA induced by destabilization of the medial meniscus in mice (34). Therefore, it was speculated that the effects of IL- 6 and IL- 8 on collagen I, MMP-1 and MMP-13 may be via STAT3 and NF- $\kappa$ B p65 signaling pathways. To confirm the hypothesis, the present study silenced the expression of STAT3 and inhibited NF- $\kappa \mathrm{B}$, using the proteasome inhibitor MG132, and then incubated chondrocytes with IL-6 or IL-8. When combined with siSTAT3 and MG132, IL-6 and IL-8 significantly reduced the levels of collagen I, MMP-1 and MMP-13, which confirmed the hypothesis. To further investigate the therapeutic mechanisms of NSAIDs and steroids in OA, piroxicam, ibuprofen, indomethacin, prednisone and betamethasone were used. No change in cell viability following treatment with these five drugs or cytokines was observed in chondrocytes compared with cells receiving no treatment. In combination with TNF- $\alpha$, prednisone, betamethasone and ibuprofen significantly reduced the expression of IL-6 and IL-8. Additionally, chondrocytes were co-cultured with drugs and IL-6 or IL-8 and it was observed that prednisone, betamethasone and ibuprofen reduced the phosphorylation of STAT3 and activation of $\mathrm{NF}-\kappa \mathrm{B}$, and also inhibited the expression of collagen I, 
MMP-1 and MMP-13. Thus, these three drugs exert a variety of protective effects in $\mathrm{OA}$.

In conclusion, the results of the present study suggest that the ability of ibuprofen, prednisone and betamethasone to protect against OA results from inhibition of IL-6 and IL-8

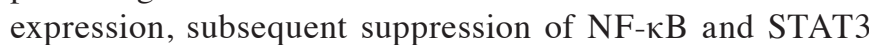
signaling pathways, and, ultimately, reduction of collagen I, MMP-1 and MMP-13 expression. The results of the present study might provide an experimental basis for the treatment of OA and guide the clinical use of drugs including ibuprofen, prednisone and betamethasone.

\section{References}

1. Cross M, Smith E, Hoy D, Nolte S, Ackerman I, Fransen M, Bridgett L, Williams S, Guillemin F, Hill CL, et al: The global burden of hip and knee osteoarthritis: Estimates from the global burden of disease 2010 study. Ann Rheum Dis 73: 1323-1330, 2014

2. Hunter DJ, Schofield D and Callander E: The individual and socioeconomic impact of osteoarthritis. Nat Rev Rheumatol 10: 437-441, 2014.

3. Felson DT: Clinical practice. Osteoarthritis of the knee. N Engl J Med 354: 841-848, 2006.

4. Goldring MB and Otero M: Inflammation in osteoarthritis. Curr Opin Rheumatol 23: 471-478, 2011.

5. Kapoor M, Martel-Pelletier J, Lajeunesse D, Pelletier JP and Fahmi H: Role of proinflammatory cytokines in the pathophysiology of osteoarthritis. Nat Rev Rheumatol 7: 33-42,2011.

6. Martel-Pelletier J: Pathophysiology of osteoarthritis. Osteoarthritis Cartilage 12 (Suppl A): S31-S33, 2004.

7. Saklatvala J: Tumour necrosis factor alpha stimulates resorption and inhibits synthesis of proteoglycan in cartilage. Nature 322: 547-549, 1986.

8. Goldring MB, Fukuo K, Birkhead JR, Dudek E and Sandell LJ: Transcriptional suppression by interleukin-1 and interferon-gamma of type II collagen gene expression in human chondrocytes. J Cell Biochem 54: 85-99, 1994.

9. Richardson DW and Dodge GR: Effects of interleukin-1beta and tumor necrosis factor-alpha on expression of matrix-related genes by cultured equine articular chondrocytes. Am J Vet Res 61: 624-630, 2000.

10. Cruz R, Miranda-Sánchez M, Solís-García D and B Kouri J: Recent patents on metalloproteinases as biomarkers in osteoarthritis diagnosis and treatment. Recent Patents on Biomarkers 4: $1-10,2014$.

11. Müller RD, John T, Kohl B, Oberholzer A, Gust T, Hostmann A, Hellmuth M, Laface D, Hutchins B, Laube G, et al: IL-10 overexpression differentially affects cartilage matrix gene expression in response to TNF-alpha in human articular chondrocytes in vitro. Cytokine 44: 377-385, 2008.

12. Jansen NW, Roosendaal G, Hooiveld MJ, Bijlsma JW, van Roon JA, Theobald M and Lafeber FP: Interleukin-10 protects against blood-induced joint damage. Br J Haematol 142: 953-961, 2008.

13. Wang Y and Lou S: Direct protective effect of interleukin-10 on articular chondrocytes in vitro. Chin Med J (Engl) 114: 723-725, 2001.

14. Fernandes JC, Martel-Pelletier J and Pelletier JP: The role of cytokines in osteoarthritis pathophysiology. Biorheology 39: 237-246, 2002

15. Borzi RM, Mazzetti I, Marcu KB and Facchini A: Chemokines in cartilage degradation. Clin Orthop Relat Res 427: S53-S61, 2004.

16. Cho H, Lee S, Park SH, Huang J, Hasty KA and Kim SJ: Synergistic effect of combined growth factors in porcine intervertebral disc degeneration. Connect Tissue Res 54: 181-186, 2013.

17. Cho H, Walker A, Williams J and Hasty KA: Study of osteoarthritis treatment with anti-inflammatory drugs: Cyclooxygenase-2 inhibitor and steroids. Biomed Res Int 2015: 595273, 2015.
18. Sokolove $\mathrm{J}$ and Lepus CM: Role of inflammation in the pathogenesis of osteoarthritis: Latest findings and interpretations. Ther Adv Musculoskelet Dis 5: 77-94, 2013.

19. Guerne PA, Carson DA and Lotz M: IL-6 production by human articular chondrocytes. Modulation of its synthesis by cytokines, growth factors, and hormones in vitro. J Immunol 144: 499-505, 1990.

20. Lotz M, Terkeltaub R and Villiger PM: Cartilage and joint inflammation. Regulation of IL-8 expression by human articular chondrocytes. J Immunol 148: 466-473, 1992.

21. Mrosewski I, Jork N, Gorte K, Conrad C, Wiegand E, Kohl B, Ertel W, John T, Oberholzer A, Kaps C and Schulze-Tanzil G: Regulation of osteoarthritis-associated key mediators by $\mathrm{TNFa}$ and IL-10: Effects of IL-10 overexpression in human synovial fibroblasts and a synovial cell line. Cell Tissue Res 357: 207-223, 2014.

22. Kaneko S, Satoh T, Chiba J, Ju C, Inoue K and Kagawa J: Interleukin-6 and interleukin-8 levels in serum and synovial fluid of patients with osteoarthritis. Cytokines Cell Mol Ther 6: 71-79, 2000.

23. Valcamonica E, Chighizola CB, Comi D, De Lucia O, Pisoni L, Murgo A, Salvi V, Sozzani S and Meroni PL: Levels of chemerin and interleukin 8 in the synovial fluid of patients with inflammatory arthritides and osteoarthritis. Clin Exp Rheumatol 32: 243-250, 2014.

24. Rowan AD, Koshy PJ, Shingleton WD, Degnan BA, Heath JK, Vernallis AB, Spaull JR, Life PF, Hudson K and Cawston TE: Synergistic effects of glycoprotein 130 binding cytokines in combination with interleukin-1 on cartilage collagen breakdown. Arthritis Rheum 44: 1620-1632, 2001.

25. Poree B, Kypriotou M, Chadjichristos C, Beauchef G, Renard E, Legendre F, Melin M, Gueret S, Hartmann DJ, Malléin-Gerin F, et al: Interleukin-6 (IL-6) and/or soluble IL-6 receptor down-regulation of human type II collagen gene expression in articular chondrocytes requires a decrease of Sp1.Sp3 ratio and of the binding activity of both factors to the COL2A1 promoter. J Biol Chem 283: 4850-4865, 2008.

26. Malemud CJ: Anticytokine therapy for osteoarthritis: Evidence to date. Drugs Aging 27: 95-115, 2010

27. Goldring MB: The role of cytokines as inflammatory mediators in osteoarthritis: Lessons from animal models. Connect Tissue Res 40: 1-11, 1999.

28. Matsukawa A, Yoshimura T, Maeda T, Ohkawara S, Takagi K and Yoshinaga M: Neutrophil accumulation and activation by homologous IL-8 in rabbits. IL-8 induces destruction of cartilage and production of IL-1 and IL-1 receptor antagonist in vivo. J Immunol 154: 5418-5425, 1995.

29. Olson TS and Ley K: Chemokines and chemokine receptors in leukocyte trafficking. Am J Physiol Regul Integr Comp Physiol 283: R7-R28, 2002.

30. Borzi RM, Mazzetti I, Magagnoli G, Paoletti S, Uguccioni M, Gatti R, Orlandini G, Cattini L and Facchini A: Growth-related oncogene alpha induction of apoptosis in osteoarthritis chondrocytes. Arthritis Rheum 46: 3201-3211, 2002.

31. OlivottoE,OteroM,MarcuKB and Goldring MB:Pathophysiology of osteoarthritis: Canonical NF- $\kappa \mathrm{B} / \mathrm{IKK} \beta$-dependent and kinase-independent effects of IKK $\alpha$ in cartilage degradation and chondrocyte differentiation. RMD Open 1 (Suppl 1): e000061, 2015.

32. Hayashi S, Nishiyama T, Hashimoto S, Fujishiro T, Kanzaki N, Iwasa K, Sakata S, Chinzei N, Kuroda R and Kurosaka M: P21 regulates MMP-13 expression through STAT3 signaling in chondrocytes. Osteoarthritis and Cartilage 21 (Suppl): S46-S47, 2013.

33. Sultana F and Rasool M: A novel therapeutic approach targeting rheumatoid arthritis by combined administration of morin, a dietary flavanol and non-steroidal anti-inflammatory drug indomethacin with reference to pro-inflammatory cytokines, inflammatory enzymes, RANKL and transcription factors. Chem Biol Interact 230: 58-70, 2015.

34. Latourte A, Cherifi C, Korng EH, Bouaziz W, Brentano TF, Cohen-Solal M, HaÿE and Richette P: Inhibition of the interleukin-6-induced STAT3 signalling pathway is chondroprotective. ECTS-IBMS, 2015. 